\title{
A Symbolic Calculus and a Parametrix Construction for Pseudodifferential Operators with Non-Smooth Negative Definite Symbols
}

\author{
Alexander Potrykus \\ Department of Mathematics \\ Swansea University \\ Singleton Park, Swansea \\ SA2 8PP - United Kingdom \\ a.k.k.potrykus@swansea.ac.uk
}

Received: February 11, 2008

Accepted: August 20, 2008

\begin{abstract}
We consider pseudodifferential operators that have non-smooth negative definite symbols and develop a corresponding symbolic calculus. Combining this symbolic calculus with the use of non-smooth symbols that are asymptotically constant in the co-variable we succeed in finding a parametrix for a certain pseudodifferential equation. This in turn allows us to show that some pseudodifferential operators with non-smooth negative definite symbols are pregenerators of Feller semigroups.
\end{abstract}

Key words: symbolic calculus, non-smooth symbols, negative definite functions, Feller semigroups.

2000 Mathematics Subject Classification: 47D06, 47G30, 35S05, 60J35, 47A53.

\section{Introduction}

Pseudodifferential operators with smooth negative definite symbols have for some time been investigated as pregenerators of Markov processes. An extensive summary of this topic including lists of references can be found in N. Jacob [10-12]. In order to see the connection between pseudodifferential operators and Markov processes, 
the key observation is that every Lévy process $\left(X_{t}\right)_{t \geq 0}$ with state space $\mathbb{R}^{n}$ is uniquely determined by a function $\psi: \mathbb{R}^{n} \rightarrow \mathbb{C}$ which is defined by

$$
E\left(e^{i X_{t} \cdot \xi}\right)=e^{-t \psi(\xi)} .
$$

The function $\psi$ is called the characteristic exponent of $\left(X_{t}\right)_{t>0}$ and is a continuous negative definite function. The prime example is Brownian motion in which case $\psi(\xi)=|\xi|^{2}$. We will refer to this as the classical case.

Furthermore, following N. Jacob [9], see also R. Schilling [15], we have for a reasonably nice Feller process $\left(\left(X_{t}\right)_{t \geq 0}, P^{x}\right)_{x \in \mathbb{R}^{n}}$ that

$$
-q(x, \xi)=\lim _{t \rightarrow 0} \frac{E^{x}\left(e^{i\left(X_{t}-x\right) \cdot \xi}\right)-1}{t}
$$

is the symbol of the pregenerator of the semigroup

$$
T_{t} u(x)=E^{x}\left(u\left(X_{t}\right)\right),
$$

i.e., on $C_{0}^{\infty}\left(\mathbb{R}^{n}\right)$,

$$
A u(x)=-q(x, D) u(x)=-(2 \pi)^{-\frac{n}{2}} \int_{\mathbb{R}^{n}} e^{i x \cdot \xi} q(x, \xi) \hat{u}(\xi) \mathrm{d} \xi .
$$

Here, $\xi \mapsto q(x, \xi)$ is for every $x \in \mathbb{R}^{n}$ a continuous negative definite function and the operator $q(x, D)$ is called a pseudodifferential operator with negative definite symbol.

W. Hoh showed in [6] how to construct Feller semigroups that are pregenerated by pseudodifferential operators with smooth negative definite symbols. His symbol classes of order $m \in \mathbb{R}$ consist of all smooth functions $q: \mathbb{R}^{n} \times \mathbb{R}^{n} \rightarrow \mathbb{C}$ such that

$$
\left|\partial_{x}^{\beta} \partial_{\xi}^{\alpha} q(x, \xi)\right| \leq c_{\alpha, \beta}(1+\psi(\xi))^{\frac{m-\rho(|\alpha|)}{2}}
$$

for all $\alpha, \beta \in \mathbb{N}_{0}^{n}$, where $\rho(k):=\min \{2,|\alpha|\}$. The function $\rho$ in this context means that higher derivatives with respect to $\xi \in \mathbb{R}^{n}$ of the symbol do not have an increasingly faster decay at infinity. The decay only improves up to order two. This behavior is typical for negative definite functions (compare, e.g., N. Jacob [11]). W. Hoh's construction of Feller semigroups is based upon the Hille-Yosida theorem, which gives three conditions that an operator $A$ has to satisfy in order to be the pregenerator of a strongly continuous contraction semigroup $\left(T_{t}\right)_{t \geq 0}$ on $C_{\infty}\left(\mathbb{R}^{n}\right)$ : The first condition is that the domain of $A$ is dense in $C_{\infty}\left(\mathbb{R}^{n}\right)$, and the second one requires that $A$ is dissipative. Both are usually easy to verify since they follow directly from properties of negative definite functions. The third condition states that the range of $\lambda-A$ is dense in $C_{\infty}\left(\mathbb{R}^{n}\right)$ for some $\lambda>0$, and is the thoughest one to check. Assuming that we have some nice embedding results at our disposal this means that we have 
to solve a pseudodifferential equation in a certain Hilbert space setting. This is usually done by constructing a parametrix to the operator $\lambda-A$. W. Hoh developed standard tools from the classical theory also for the negative definite symbols case, e.g., boundedness of pseudodifferential operators in scales of (anisotropic) Sobolev spaces, (sharp) Gårding inequality, Friedrichs symmetrization, etc., and successfully constructed Feller semigroups using the Hille-Yosida theorem.

Let us go back for a moment to our original motivation in studying pseudodifferential operators with negative definite symbols. One of the standard examples aside from Brownian motion is the Cauchy process. In the space-invariant setting it is easy to see that the semigroup corresponding to the Cauchy process is pregenerated by a pseudodifferential operator with continuous negative definite symbol $\xi \mapsto|\xi|$. Now we make this symbol space-dependent, e.g., we consider a pseudodifferential operator with symbol $p: \mathbb{R}^{n} \rightarrow \mathbb{R}^{n} \rightarrow \mathbb{R},(x, \xi) \mapsto a(x)|\xi|$, where $a: \mathbb{R}^{n} \rightarrow \mathbb{R}$ is a smooth function. Following W. Hoh's approach of construction a corresponding Feller process using the Hille-Yosida theorem, we first of all note that this symbol is not even differentiable in the $\xi$-variable, i.e., it does not belong to any of W. Hoh's symbol classes. On the other hand, one could try the naive approach of constructing a parametrix for pseudodifferential operators with non-smooth symbols and ask the question how much differentiability the symbols would (at least) be expected to have. First of all, parametrix constructions use asymptotic expansions up to first order of the involved symbol, i.e., the symbol would need to be at least differentiable up to order one in both variables. Also, since we in addition need to use embedding results, we should expect to have certain restrictions imposed on the differentiability with respect to $x \in \mathbb{R}^{n}$. All of this tells us that by following W. Hoh's approach and using the Hille-Yosida theorem, we will not be able to cover the above-mentioned case involving the state-space dependent version of the Cauchy process. Nevertheless, we find at the end of section 2 that it is possible to construct Feller processes starting with pseudodifferential operators that have negative definite symbols with sufficiently high (but non-smooth) differentiability.

In section 1 we begin by formally introducing negative definite functions and some of the associated concepts. The main part of this section is dedicated to the symbolic calculus for non-smooth negative definite symbols.

Section 2 introduces a subclass of our non-smooth negative definite symbols, which has the additional property that the symbols are asymptotically constant in the co-variable. This technique as well as the part of Fredholm theory we use can be found for classical symbol classes in H. Kumano-go [13] or V. V. Grushin [5]. We then proceed with using our symbolic calculus developed in section 2 to construct a parametrix and in the end a Feller semigroup. 


\section{Negative definite functions and symbolic calculus}

Let us begin with the fundamental notion of this paper - negative definite functions. The following theorem contains the Lévy-Khinchin formula which identifies all such functions.

Theorem 1.1. Let $\psi: \mathbb{R}^{n} \rightarrow \mathbb{C}$ be a continuous negative definite function. Then there exists a constant $c \geq 0$, a vector $d \in \mathbb{R}^{n}$, a symmetric positive semidefinite quadratic form $q$ on $\mathbb{R}^{n}$ and a finite measure $\mu$ on $\mathbb{R}^{n} \backslash\{0\}$ such that

$$
\psi(\xi)=c+i(d \cdot \xi)+q(\xi)+\int_{\mathbb{R}^{n} \backslash\{0\}}\left(1-e^{-i x \cdot \xi}-\frac{i x \cdot \xi}{1+|x|^{2}}\right) \frac{1+|x|^{2}}{|x|^{2}} \mu(\mathrm{d} x)
$$

holds.

For real-valued continuous negative definite functions we may state more explicitly

Corollary 1.2. Let $\psi: \mathbb{R}^{n} \rightarrow \mathbb{R}$ be a real-valued continuous negative definite function. Then we have the representation

$$
\psi(\xi)=c+q(\xi)+\int_{\mathbb{R}^{n} \backslash\{0\}}(1-\cos (x \cdot \xi)) \frac{1+|x|^{2}}{|x|^{2}} \mu(\mathrm{d} x)
$$

with $c, q, \mu$ as in Theorem 1.1. In addition $\mu$ is a symmetric measure.

Instead of working with $\mu$ we often consider the Lévy measure associated with $\psi$, i.e.,

$$
\nu(\mathrm{d} x):=\frac{1+|x|^{2}}{|x|^{2}} \mu(\mathrm{d} x) .
$$

Thus $\nu$ satisfies the integrability condition $\int_{\mathbb{R}^{n} \backslash\{0\}}\left(|x|^{2} \wedge 1\right) \nu(\mathrm{d} x)<\infty$.

The following two results are due to W. Hoh [6]. The first relates the smoothness of $\psi$ to integrability properties of $\nu$.

Theorem 1.3. Let $\psi: \mathbb{R}^{n} \rightarrow \mathbb{R}$ be a continuous negative definite function with LévyKhinchin representation as in Corollary 1.2. Suppose that for $2 \leq l \leq k$ all absolute moments of the Lévy measure $\nu$ exist, i.e.,

$$
M_{l}:=\int_{\mathbb{R}^{n} \backslash\{0\}}|x|^{l} \nu(\mathrm{d} x)<\infty, \quad 2 \leq l \leq k .
$$

Then $\psi$ is of class $C^{k}\left(\mathbb{R}^{n}\right)$ and for $\alpha \in \mathbb{N}_{0}^{n},|\alpha| \leq k$, we have the estimate

$$
\left|\partial_{\xi}^{\alpha} \psi(\xi)\right| \leq c_{|\alpha|} \begin{cases}\psi(\xi), & \alpha=0 \\ \psi(\xi)^{\frac{1}{2}}, & |\alpha|=1, \\ 1, & |\alpha| \geq 2,\end{cases}
$$

with $c_{0}=1, c_{1}=\left(2 M_{2}\right)^{\frac{1}{2}}+2 \lambda^{\frac{1}{2}}, c_{2}=M_{2}+2 \lambda$, and $c_{l}=M_{l}$ for $3 \leq l \leq k$, where $\lambda$ is the maximal eigenvalue of the quadratic form $q$ in Corollary 1.2. 
The next Lemma enables us to formulate in a sensible way symbol classes, compare for this topic also W. Hoh [6].

Lemma 1.4. Suppose that the continuous negative definite function $\psi: \mathbb{R}^{n} \rightarrow \mathbb{R}$ satisfies (1) for all $k \in \mathbb{N}$. Then for all $m \in \mathbb{R}$ and all $\alpha \in \mathbb{N}_{0}^{n}$ we have

$$
\left|\partial_{\xi}^{\alpha}(1+\psi(\xi))^{\frac{m}{2}}\right| \leq c_{|\alpha|}(1+\psi(\xi))^{\frac{m-\rho(|\alpha|)}{2}}
$$

where $\rho(k):=\min \{k, 2\}$.

Definition 1.5. A continuous negative definite function $\psi: \mathbb{R}^{n} \rightarrow \mathbb{R}$ belongs to class $\Lambda^{\infty}$ if it satisfies (2).

Let us moreover mention here that negative definite functions have at most quadratic growth, i.e.,

Lemma 1.6. If $\psi: \mathbb{R}^{n} \rightarrow \mathbb{C}$ is a continuous negative definite function then

$$
|\psi(\xi)| \leq c_{\psi}\left(1+|\xi|^{2}\right)
$$

for $\xi \in \mathbb{R}^{n}$ where $c_{\psi}=2 \sup _{|\eta| \leq 1}|\psi(\eta)|$.

Since we want to work with non-smooth symbols let us introduce some notations: We denote from now on by $C^{M_{x}, M_{\xi}}\left(\mathbb{R}^{n} \times \mathbb{R}^{n}\right), M_{x}, M_{\xi} \in \mathbb{N}_{0} \cup\{\infty\}$, the space of all functions $p: \mathbb{R}^{n} \times \mathbb{R}^{n} \rightarrow \mathbb{R},(x, \xi) \mapsto p(x, \xi)$, such that for all $\alpha, \beta \in \mathbb{N}_{0}^{n}$ with $|\alpha| \leq M_{\xi}$, and $|\beta| \leq M_{x}$, the derivatives $\partial_{\xi}^{\alpha} p(x, \xi)$ and $\partial_{x}^{\beta} p(x, \xi)$ are continuous. We can now give the definition of symbol classes of non-smooth negative definite functions.

Definition 1.7. Let $\psi \in \Lambda^{\infty}, m \in \mathbb{R}$, and $p: \mathbb{R}^{n} \times \mathbb{R}^{n} \rightarrow \mathbb{C}$ be a $C^{M_{x}, M_{\xi}}\left(\mathbb{R}^{2 n}\right)$ function where $M_{x}, M_{\xi} \in \mathbb{N}_{0} \cup\{\infty\}$. We say that $p$ is a symbol of order $m$, and write $p \in S_{\rho_{j}}^{m, \psi}\left(M_{x}, M_{\xi}\right)$, if for all multiindices $\alpha, \beta \in \mathbb{N}_{0}^{n}$ with $|\alpha| \leq M_{\xi}$ and $|\beta| \leq M_{x}$ there exist constants $c_{\alpha, \beta}$ such that

$$
\left|\partial_{\xi}^{\alpha} \partial_{x}^{\beta} p(x, \xi)\right| \leq c_{\alpha, \beta}(1+\psi(\xi))^{\frac{m-\rho_{j}(|\alpha|)}{2}}
$$

where $\rho_{j}(k)=\min \{k, j\}$ and $j \in\{0,1,2\}$.

Let us give a remark concerning the role of the function $\rho_{j}$ : Let $p \in S_{\rho_{2}}^{m, \psi}$ be given, then $\partial_{\xi}^{\alpha} p \in S_{\rho_{1}}^{m-1, \psi}$ for $|\alpha|=1$ and $\partial_{\xi}^{\alpha} p \in S_{\rho_{0}}^{m-2, \psi}$ for $|\alpha|=2$. Note that $\rho_{0} \equiv 0$. This notation will be useful when introducing asymptotic expansions later on. Using (3) we can also define a family of seminorms on $S_{\rho_{j}}^{m, \psi}$ :

$$
|p|_{k}^{(m)}:=\max _{|\alpha|,|\beta| \leq k} \sup _{x, \xi \in \mathbb{R}^{n}}\left|\partial_{\xi}^{\alpha} \partial_{x}^{\beta} p(x, \xi)\right|(1+\psi(\xi))^{-\frac{m}{2}},
$$

where $k \leq \min \left\{M_{x}, M_{\xi}\right\}$.

Since we will use oscillatory integrals to express the symbol of the composition of two pseudodifferential operators we also need to modify the notion of amplitudes to fit in with our non-smooth setting. 
Definition 1.8. The space of amplitudes $A_{M_{y}, M_{\eta}}^{m}\left(\mathbb{R}^{n} \times \mathbb{R}^{n}\right), m \geq 0$, is the set of all $C^{M_{y}, M_{\eta}}\left(\mathbb{R}^{n}, \mathbb{R}^{n}\right)$ differentiable functions such that

$$
\left|\partial_{\eta}^{\alpha} \partial_{y}^{\beta} a(y, \eta)\right| \leq c_{\alpha, \beta}\left(1+|\eta|^{2}\right)^{\frac{m}{2}}\left(1+|y|^{2}\right)^{\frac{m}{2}}
$$

uniformly in $y, \eta \in \mathbb{R}^{n}$ for all $\alpha, \beta \in \mathbb{N}_{0}^{n}$ such that $|\alpha| \leq M_{\eta},|\beta| \leq M_{y}$. On the space $A_{M_{y}, M_{\eta}}^{m}$ we use the seminorms defined by

$$
\|a\|_{k}:=\max _{|\alpha|+|\beta| \leq k} \sup _{y, \eta \in \mathbb{R}^{n}}\left(1+|\eta|^{2}\right)^{-\frac{m}{2}}\left(1+|y|^{2}\right)^{-\frac{m}{2}}\left|\partial_{\eta}^{\alpha} \partial_{y}^{\beta} a(y, \eta)\right|,
$$

where $m$ is fixed and $k \leq \min \left\{M_{y}, M_{\eta}\right\}$.

Note that Definition 1.8 is similar to the definition of amplitudes in the smooth case, compare W. Hoh [6], N. Jacob [11], or H. Kumano-go [13]. The following is a standard theorem used for estimating oscillatory integrals and can be found in, e.g., S. Alinhac and P. Gérard [1] or H. Kumano-go [13]. In its original form the theorem tells us for smooth amplitudes $a \in A_{\infty, \infty}^{m}$ we can estimate the corresponding oscillatory integral with

$$
\mid \text { Os- } \iint_{\mathbb{R}^{2 n}} e^{i y \cdot \eta} a(y, \eta) \mathrm{d} y \mathrm{~d} \eta \mid \leq C_{m}\|a\|_{m+2 n+1} .
$$

This statement leads us to suspect that (4) should also hold for amplitudes of class $A_{M_{y}, M_{\eta}}^{m}$ where $M_{y} \geq m+2 n+1$ and $M_{\eta} \geq m+2 n+1$. Indeed this is easily verified by looking at the proof. Hence we may state

Theorem 1.9. If $a \in A_{M_{y}, M_{\eta}}^{m}, m \geq 0, M_{y} \geq m+2 n+1, M_{\eta} \geq m+2 n+1$, then

$$
\mid \text { Os- } \iint_{\mathbb{R}^{2 n}} e^{i y \cdot \eta} a(y, \eta) \mathrm{d} y \mathrm{~d} \eta \mid \leq C_{m}\|a\|_{m+2 n+1} .
$$

These are all the prerequisites we need in order to develop our theory. Since our aim is to work with non-smooth symbols let us from now on assume that we are given for $\psi \in \Lambda^{\infty}$,

$$
\begin{aligned}
& p \in S_{\rho_{2}}^{m, \psi}\left(M_{x}^{p}, M_{\xi}^{p}\right), \\
& q \in S_{\rho_{2}}^{l, \psi}\left(M_{x}^{q}, M_{\xi}^{q}\right),
\end{aligned}
$$

where $m, l \in \mathbb{R}$ and $M_{x}^{p}, M_{\xi}^{p}, M_{x}^{q}, M_{\xi}^{q} \in \mathbb{N}_{0} \cup\{\infty\}$. Our first step is to look at the symbol of the composition of the two pseudodifferential operators $p(x, D)$ and $q(x, D)$. Assuming for the moment that our symbols $p$ and $q$ are smooth, i.e., that $M_{x}^{p}=M_{\xi}^{p}=$ $M_{x}^{q}=M_{\xi}^{q}=\infty$, it is a well-known fact that the symbol $(p \circ q)$ of the composition of both operators can be written using oscillatory integrals as

$$
(p \circ q)(x, \xi)=(2 \pi)^{-n} \mathrm{Os}-\iint_{\mathbb{R}^{2 n}} e^{-i y \cdot \eta} p(x, \xi-\eta) q(x-y, \xi) \mathrm{d} y \mathrm{~d} \eta .
$$


The calculation leading to (5) is straightforward, one only needs to pay attention to whether the oscillatory integrals exists. But this can be checked using (4). In the same way, we might use (5) together with Theorem 1.9 as a starting point when considering the non-smooth case.

Before continuing we would like to point out that our aim is not only to show that $p \circ q$ is again a non-smooth symbol of order $m+l$, but also to develop an asymptotic expansion formula. This is needed for the parametrix construction in the next section. Note that this essentially means that we need to show that

$$
(p \circ q)(x, \xi)=p(x, \xi) q(x, \xi)+r(x, \xi)
$$

where $r$ is a symbol of order $m+l-1$. Since $p q$ is a symbol of order $m+l$ this also gives us that $(p \circ q)$ is of order $m+l$. Of course the differentiability of each symbol also needs to be investigated. Note that in this respect, (6) poses an interesting question. In the (smooth) classical symbolic calculus one can find an asymptotic expansion for the symbol of the composition of two pseudodifferential operators where the order of each successive term decreases by one. In case of W. Hoh's symbolic calculus, one only finds improvement in terms of order up to the third term of the expansion. As explained earlier this is due to properties of negative definite functions. In our case, where we additionally need to take care of the differentiability conditions on our symbols, we expect to see a difference in terms of required differentiability when considering the expansions

$$
(p \circ q)(x, \xi)=p(x, \xi) q(x, \xi)+r_{1}(x, \xi)
$$

and

$$
(p \circ q)(x, \xi)=p(x, \xi) q(x, \xi)+\sum_{|\alpha|=1} \partial_{\xi}^{\alpha} p(x, \xi) D_{x}^{\alpha} q(x, \xi)+r_{2}(x, \xi)
$$

where $D_{x}^{\alpha}=(-i)^{|\alpha|} \partial_{x}^{\alpha}$. If we would want to work with (8) we surely needed smoother symbols than for (7). On the other hand, if we do not need asymptotic expansions, but want only to work with the symbol $(p \circ q)$, we could do with less smooth symbols. Of course, for parametrix constructions, (7) suffices. Nevertheless we will consider all three cases.

Let us take a look at a simple example: Set $n=1$ and consider the Laplace operator $\Delta_{x}=\frac{\mathrm{d}^{2}}{\mathrm{~d} x^{2}}$ that has symbol $-\xi^{2}$. Set $\psi(\xi):=\xi^{2}$ and note that this is a continuous negative definite function of class $\Lambda^{\infty}$. Also, let $q \in S_{\rho_{2}}^{2, \psi}\left(M_{x}^{q}, M_{\xi}^{q}\right)$ be given where $q(x, \xi)=a(x) \tilde{q}(\xi)$, i.e., $a \in C^{M_{x}^{q}}(\mathbb{R}), \tilde{q} \in C^{M_{\xi}^{q}}(\mathbb{R})$. Then

$$
\begin{aligned}
\Delta_{x} q(x, D) u(x) & =(2 \pi)^{-\frac{n}{2}} \int_{\mathbb{R}} \sum_{r=0}^{2}\left(\begin{array}{l}
2 \\
r
\end{array}\right)\left(\frac{\mathrm{d}^{r}}{\mathrm{~d} x^{r}} e^{i x \xi}\right)\left(\frac{\mathrm{d}^{2-r}}{\mathrm{~d} x^{2-r}} q(x, \xi)\right) \tilde{u}(\xi) \mathrm{d} \xi \\
& =(2 \pi)^{-\frac{n}{2}} \int_{\mathbb{R}^{n}} e^{i x \xi} \sum_{r=0}^{2}\left(\begin{array}{l}
2 \\
r
\end{array}\right) i^{r} \xi^{r}\left(\frac{\mathrm{d}^{2-r}}{\mathrm{~d} x^{2-r}} a(x)\right) \tilde{q}(\xi) \tilde{u}(\xi) \mathrm{d} \xi,
\end{aligned}
$$


i.e., we need that $M_{x}^{q} \geq 2$. Obviously, if we consider $\Delta_{x}^{m}$ for $m \in \mathbb{N}$ instead of $\Delta_{x}$, then we need $M_{x}^{q} \geq 2 m$. This leads us to suspect that if we consider the composition $p(x, D) \circ q(x, D)$ of two symbols $p \in S_{\rho_{2}}^{m, \psi}\left(M_{x}^{p}, M_{\xi}^{p}\right)$ and $q \in S_{\rho_{2}}^{l, \psi}\left(M_{x}^{q}, M_{\xi}^{q}\right)$ then $M_{x}^{q}$ should depend on the order $m$ of $p$.

Another important example to consider is the case where $p \in S_{\rho_{2}}^{m, \psi}\left(M_{x}^{p}, M_{\xi}^{p}\right)$ and $q \in S_{\rho_{2}}^{m, \psi}\left(M_{x}^{p}, M_{\xi}^{p}\right)$ such that $q(x, \xi)=q(\xi)$. In this case it is easy to see that

$$
p(x, D) \circ q(D) u(x)=(2 \pi)^{-\frac{n}{2}} \int_{\mathbb{R}^{n}} e^{i x \cdot \xi} p(x, \xi) q(\xi) \hat{u}(\xi) \mathrm{d} \xi,
$$

i.e., the symbol of the composition is just the product of the symbols. Clearly no restrictions on the differentiability of $p$ and $q$ are needed.

Theorem 1.10. Let $\psi \in \Lambda^{\infty}, p \in S_{\rho_{j}}^{m, \psi}\left(M_{x}^{p}, M_{\xi}^{p}\right)$ and $q \in S_{\rho_{j}}^{l, \psi}\left(M_{x}^{q}, M_{\xi}^{q}\right)$ be given for $m, l \in \mathbb{R}, j \in\{0,1,2\}$ and $M_{x}^{p}, M_{\xi}^{p}, M_{x}^{q}, M_{\xi}^{q} \in \mathbb{N}_{0} \cup\{\infty\}$.

(i) If

$$
M_{\xi}^{p} \geq|m|+j+2 n+1 \quad \text { and } \quad M_{x}^{q} \geq|m|+j+2 n+1
$$

then

$$
(p \circ q)(x, \xi):=(2 \pi)^{-n} \mathrm{Os}-\iint_{\mathbb{R}^{2 n}} e^{-i y \cdot \eta} p(x, \xi-\eta) q(x-y, \xi) \mathrm{d} y \mathrm{~d} \eta
$$

defines a symbol of class $S_{\rho_{j}}^{m+l, \psi}\left(M_{x}^{p \circ q}, M_{\xi}^{p \circ q}\right)$ where

$$
M_{x}^{p \circ q}=\min \left\{M_{x}^{p}, M_{x}^{q}-|m|-j-2 n-1\right\}
$$

and

$$
M_{\xi}^{p \circ q}=\min \left\{M_{\xi}^{q}, M_{\xi}^{p}-|m|-j-2 n-1\right\} .
$$

(ii) If $j \in\{1,2\}$ and

$$
M_{\xi}^{p} \geq|m-1|+j+2 n+1 \quad \text { as well as } \quad M_{x}^{q} \geq|m-1|+j+2 n+1
$$

then

$$
(p \circ q)(x, \xi)=p(x, \xi) q(x, \xi)+r_{1}(x, \xi)
$$

with $r_{1} \in S_{\rho_{j-1}}^{m+l-1, \psi}\left(M_{x}^{r_{1}}, M_{\xi}^{r_{1}}\right)$ where

$$
M_{x}^{r_{1}}=\min \left\{M_{x}^{p}, M_{x}^{q}-|m-1|-j-2 n-1\right\}
$$

and

$$
M_{\xi}^{r_{2}}=\min \left\{M_{\xi}^{q}, M_{\xi}^{p}-|m-1|-j-2 n-1\right\} .
$$


(iii) If $j=2$ and

$$
M_{\xi}^{p} \geq|m-2|+j+2 n+1 \quad \text { and } \quad M_{x}^{q} \geq|m-2|+j+2 n+1
$$

then

$$
(p \circ q)(x, \xi)=p(x, \xi) q(x, \xi)+\sum_{|\alpha|=1} \partial_{\xi}^{\alpha} p(x, \xi) D_{x}^{\alpha} q(x, \xi)+r_{2}(x, \xi)
$$

with $r_{2} \in S_{\rho_{0}}^{m+l-2, \psi}\left(M_{x}^{r_{2}}, M_{\xi}^{r_{2}}\right)$ where

$$
M_{x}^{r_{2}}=\min \left\{M_{x}^{p}, M_{x}^{q}-|m-2|-j-2 n-1\right\}
$$

and

$$
M_{\xi}^{r_{2}}=\min \left\{M_{\xi}^{q}, M_{\xi}^{p}-|m-2|-j-2 n-1\right\} .
$$

Proof. (i) Note that

$$
\partial_{\xi}^{\alpha} \partial_{x}^{\beta}(p \circ q)=\sum_{\alpha^{\prime} \leq \alpha} \sum_{\beta^{\prime} \leq \beta}\left(\begin{array}{c}
\alpha \\
\alpha^{\prime}
\end{array}\right)\left(\begin{array}{c}
\beta \\
\beta^{\prime}
\end{array}\right)\left(\left(\partial_{\xi}^{\alpha^{\prime}} \partial_{x}^{\beta^{\prime}} p\right) \circ\left(\partial_{\xi}^{\alpha-\alpha^{\prime}} \partial_{x}^{\beta-\beta^{\prime}} q\right)\right) .
$$

We set

$$
c_{x, \xi}(y, \eta):=\left(\partial_{\xi}^{\alpha^{\prime}} \partial_{x}^{\beta^{\prime}} p(x, \xi-\eta)\right)\left(\partial_{\xi}^{\alpha-\alpha^{\prime}} \partial_{x}^{\beta-\beta^{\prime}} q(x-y, \xi)\right)
$$

and find using the subadditivity of $\rho_{j}$ as well as Peetre's inequality for negative definite functions (compare, e.g., Lemma 3.6.23 in N. Jacob [10]) that

$$
\begin{aligned}
\left|\partial_{y}^{\gamma} \partial_{\eta}^{\delta} c_{x, \xi}(y, \eta)\right| & \leq\left|\partial_{\eta}^{\delta} \partial_{\xi}^{\alpha^{\prime}} \partial_{x}^{\beta^{\prime}} p(x, \xi-\eta)\right|\left|\partial_{y}^{\gamma} \partial_{\xi}^{\alpha-\alpha^{\prime}} \partial_{x}^{\beta-\beta^{\prime}} q(x-y, \xi)\right| \\
& \leq c(1+\psi(\xi-\eta))^{\frac{m-\rho_{j}\left(\left|\alpha^{\prime}+\delta\right|\right)}{2}}(1+\psi(\xi))^{\frac{l-\rho_{j}\left(\left|\alpha-\alpha^{\prime}\right|\right)}{2}} \\
& \leq c^{\prime}(1+\psi(\xi))^{\frac{m+l-\rho_{j}(|\alpha+\delta|)}{2}}(1+\psi(\eta))^{\frac{|m|+\rho_{j}\left(\left|\alpha^{\prime}+\delta\right|\right)}{2}} \\
& \leq C(1+\psi(\xi))^{\frac{m+l-\rho_{j}(|\alpha|)}{2}}\left(1+|\eta|^{2}\right)^{\frac{|m|+j}{2}} .
\end{aligned}
$$

Therefore, $c_{x, \xi}$ is an amplitude of order $|m|+j$. In order to use Theorem 1.9, we need to calculate $\left\|c_{x, \xi}\right\|_{|m|+j+2 n+1}$ and determine what the minimal differentiability conditions for $p$ and $q$ are. By definition we have

$$
\begin{aligned}
& \left\|c_{x, \xi}\right\|_{|m|+j+2 n+1} \\
& \quad=\max _{|\gamma|+|\delta| \leq|m|+j+2 n+1} \sup _{y, \eta \in \mathbb{R}^{n}}\left(1+|y|^{2}\right)^{-\frac{|m|+j}{2}}\left(1+|\eta|^{2}\right)^{-\frac{|m|+j}{2}}\left|\partial_{y}^{\gamma} \partial_{\eta}^{\delta} c_{x, \xi}(y, \eta)\right|,
\end{aligned}
$$

which gives us that

$$
\left\|c_{x, \xi}\right\| \|_{|m|+j+2 n+1} \leq C\left(1+\psi\left(m+l-\rho_{j}(|\alpha|)\right)\right)^{\frac{\xi}{2}} .
$$


With regard to the differentiability conditions, we look at

$$
\left|\partial_{y}^{\gamma} \partial_{\eta}^{\delta} c_{x, \xi}(y, \eta)\right| \leq\left|\partial_{\eta}^{\delta} \partial_{\xi}^{\alpha^{\prime}} \partial_{x}^{\beta^{\prime}} p(x, \xi-\eta)\right|\left|\partial_{y}^{\gamma} \partial_{\xi}^{\alpha-\alpha^{\prime}} \partial_{x}^{\beta-\beta^{\prime}} q(x-y, \xi)\right| .
$$

Hence it is clear that the above calculations are valid if $M_{\xi}^{p} \geq|m|+j+2 n+1$ and $M_{x}^{q} \geq|m|+j+2 n+1$. Furthermore we need to restrict $\alpha, \beta \in \mathbb{N}_{0}^{n}$ so that $|\alpha| \leq \min \left\{M_{\xi}^{q}, M_{\xi}^{p}-|m|-j-2 n-1\right\}$ and $|\beta| \leq \min \left\{M_{x}^{p}, M_{x}^{q}-|m|-j-2 n-1\right\}$. Now we use Theorem 1.9 and find

$$
\left|\partial_{\xi}^{\alpha} \partial_{x}^{\beta}(p \circ q)(x, \xi)\right| \leq C\left\|c_{x, \xi}\right\|_{|m|+j+2 n+1} \leq C^{\prime}\left(1+\psi\left(m+l-\rho_{j}(|\alpha|)\right)\right)^{\frac{\xi}{2}} .
$$

(ii) First of all, we know by (9) that

$$
(p \circ q)(x, \xi)=(2 \pi)^{-n} \mathrm{Os}-\iint_{\mathbb{R}^{2 n}} e^{-i y \cdot \eta} p(x, \xi-\eta) q(x-y, \xi) \mathrm{d} y \mathrm{~d} \eta .
$$

Using Taylor's formula we write

$$
p(x, \xi-\eta)=p(x, \xi)+\sum_{|\alpha|=1}(-\eta)^{\alpha} \int_{0}^{1} \partial_{\xi}^{\alpha} p(x, \xi-t \eta) \mathrm{d} t .
$$

Now note that

$$
\begin{aligned}
(2 \pi)^{-n} \mathrm{Os}-\iint_{\mathbb{R}^{2 n}} e^{-i y \cdot \eta} p(x, \xi) q(x- & y, \xi) \mathrm{d} y \mathrm{~d} \eta \\
& =p(x, \xi)(2 \pi)^{-n} \mathrm{Os}-\iint_{\mathbb{R}^{2 n}} e^{-i y \cdot \eta} q(x-y, \xi) \mathrm{d} y \mathrm{~d} \eta \\
& =p(x, \xi) q(x-0, \xi)=p(x, \xi) q(x, \xi) .
\end{aligned}
$$

Hence we have that

$$
(p \circ q)(x, \xi)=p(x, \xi) q(x, \xi)+r_{1}(x, \xi)
$$

where

$$
\begin{aligned}
r_{1}(x, \xi) & =\sum_{|\alpha|=1}(2 \pi)^{-n} \mathrm{Os}-\iint_{\mathbb{R}^{2 n}} e^{-i y \cdot \eta}(-\eta)^{\alpha} \int_{0}^{1} \partial_{\xi}^{\alpha} p(x, \xi-t \eta) \mathrm{d} t q(x-y, \xi) \mathrm{d} y \mathrm{~d} \eta \\
& =\sum_{|\alpha|=1}(2 \pi)^{-n} \mathrm{Os}-\iint_{\mathbb{R}^{2 n}} e^{-i y \cdot \eta} \int_{0}^{1} \partial_{\xi}^{\alpha} p(x, \xi-t \eta) \mathrm{d} t\left(-D_{y}\right)^{\alpha} q(x-y, \xi) \mathrm{d} y \mathrm{~d} \eta .
\end{aligned}
$$

Then

$$
\partial_{\xi}^{\gamma} \partial_{x}^{\delta} r_{1}(x, \xi)=\sum_{|\alpha|=1} \sum_{\gamma^{\prime} \leq \gamma} \sum_{\delta^{\prime} \leq \delta}\left(\begin{array}{l}
\gamma \\
\gamma^{\prime}
\end{array}\right)\left(\begin{array}{c}
\delta \\
\delta^{\prime}
\end{array}\right)(2 \pi)^{-n} \mathrm{Os}-\iint_{\mathbb{R}^{2 n}} e^{-i y \cdot \eta} a_{r_{1}, x, \xi}(y, \eta) \mathrm{d} y \mathrm{~d} \eta
$$


where we have set

$$
a_{r_{1}, x, \xi}(y, \eta):=\int_{0}^{1} \partial_{\xi}^{\gamma^{\prime}} \partial_{x}^{\delta^{\prime}} \partial_{\xi}^{\alpha} p(x, \xi-t \eta) \mathrm{d} t \partial_{\xi}^{\gamma-\gamma^{\prime}} \partial_{x}^{\delta-\delta^{\prime}}\left(-D_{y}\right)^{\alpha} q(x-y, \xi) .
$$

Using Peetre's inequality for negative definite functions we find in a similar manner as in (i) that

$$
\begin{aligned}
& \int_{0}^{1}\left|\partial_{\xi}^{\gamma^{\prime}} \partial_{x}^{\delta^{\prime}} \partial_{\xi}^{\alpha} p(x, \xi-t \eta)\right| \mathrm{d} t\left|\partial_{\xi}^{\gamma-\gamma^{\prime}} \partial_{x}^{\delta-\delta^{\prime}}\left(-D_{y}\right)^{\alpha} q(x-y, \xi)\right| \\
& \leq c\left(1+\psi\left(m+l-1-\rho_{j-1}(|\gamma|)\right)\right)^{\frac{\xi}{2}}(1+\psi(\eta))^{\frac{|m-1|+j-1}{2}} \\
& \leq C\left(1+\psi\left(m+l-1-\rho_{j-1}(|\gamma|)\right)\right)^{\frac{\xi}{2}}\left(1+|\eta|^{2}\right)^{\frac{|m-1|+j-1}{2}}
\end{aligned}
$$

We conclude

$$
\left|a_{r_{1}, x, \xi}(y, \eta)\right| \leq C^{\prime}\left(1+\psi\left(m+l-1-\rho_{j-1}(|\gamma|)\right)\right)^{\frac{\xi}{2}}\left(1+|\eta|^{2}\right)^{\frac{|m-1|+j-1}{2}},
$$

i.e., $a_{r_{1}, x, \xi}$ is an amplitude of order $|m-1|+j-1$. We hence find

$$
\left\|\left|a_{r_{1}}\right|\right\|_{|m-1|+j-1+2 n+1}=\left\|a_{r_{1}}\right\|_{|m-1|+j+2 n} \leq c\left(1+\psi\left(m+l-1-\rho_{j-1}(|\gamma|)\right)\right)^{\frac{\xi}{2}}
$$

which together with Theorem 1.9 yields

$$
\left|\partial_{\xi}^{\gamma} \partial_{x}^{\delta} r_{1}(x, \xi)\right| \leq C \mid\left\|a_{r_{1}}\right\|_{|m-1|+j+2 n} \leq c\left(1+\psi\left(m+l-1-\rho_{j-1}(|\gamma|)\right)\right)^{\frac{\xi}{2}} .
$$

This means that $r_{1} \in S_{\rho_{j-1}}^{m+l-1, \psi}$. It remains to find the differentiability conditions. As in (i) we find that the above calculations are valid as long as $M_{\xi}^{p} \geq|m-1|+j+2 n+1$ and $M_{x}^{q} \geq|m-1|+j+2 n+1$. Furthermore we need to restrict $\gamma, \delta \in \mathbb{N}_{0}^{n}$ to $|\gamma| \leq \min \left\{M_{\xi}^{q}, M_{\xi}^{p}-|m-1|-j-2 n-1\right\}$ and $|\delta| \leq \min \left\{M_{x}^{p}, M_{x}^{q}-|m-1|-j-2 n-1\right\}$.

(iii) This follows from the same reasoning as in (ii). Here, we use use the Taylor expansion

$$
p(x, \xi-\eta)=p(x, \xi)+\sum_{|\alpha|=1}(-\eta)^{\alpha} \partial_{\xi}^{\alpha} p(x, \xi)+\sum_{|\alpha|=2}(-\eta)^{\alpha} \int_{0}^{1}(1-t) \partial_{\xi}^{\alpha} p(x, \xi-t \eta) \mathrm{d} t .
$$

The first term of the expansion then follows as in (ii). For the second term we calculate

$$
\begin{aligned}
\sum_{|\alpha|=1}(2 \pi)^{-n} \text { Os }-\iint_{\mathbb{R}^{2 n}} & e^{-i y \cdot \eta}(-\eta)^{\alpha} \partial_{\xi}^{\alpha} p(x, \xi) q(x-y, \xi) \mathrm{d} y \mathrm{~d} \eta \\
& =\sum_{|\alpha|=1} \partial_{\xi}^{\alpha} p(x, \xi)(2 \pi)^{-n} \text { Os- } \iint_{\mathbb{R}^{2 n}} e^{-i y \cdot \eta}\left(D_{y}\right)^{\alpha} q(x-y, \xi) \mathrm{d} y \mathrm{~d} \eta \\
& =\sum_{|\alpha|=1} \partial_{\xi}^{\alpha} p(x, \xi) D_{x}^{\alpha} q(x, \xi) .
\end{aligned}
$$


We next consider the remainder term $r_{2}$ given by

$$
r_{2}(x, \xi)=\sum_{|\alpha|=2}(2 \pi)^{-n} \text { Os- } \iint_{\mathbb{R}^{2 n}}(-\eta)^{\alpha} \int_{0}^{1}(1-t) \partial_{\xi}^{\alpha} p(x, \xi-t \eta) \mathrm{d} t q(x-y, \xi) \mathrm{d} y \mathrm{~d} \eta
$$

The proof from here on is basically identical to the one for (ii). All that remains to be done is to set $|\alpha|=2$ and use the function $\rho_{j-2}$ instead of $\rho_{j-1}$ as done in (ii).

Note that this theorem coincides with our observations made earlier on. The minimum differentiability that the symbol $q$ needs to have with respect to $x$ depends on the order of the symbol $p$ ! In terms of embedding results which we need in the next section this means that we expect to choose $M_{x}^{q}$ very large. Also we emphasize that we do not mention the symbol of the adjoint of a pseudodifferential operator within our symbolic calculus for non-smooth symbols. One might argue that the symbol of the adjoint is given by the oscillatory integral

$$
(2 \pi)^{-n} \text { Os- } \iint_{\mathbb{R}^{2 n}} e^{-i y \cdot \eta} \overline{p(x-y, \xi-\eta)} \mathrm{d} y \mathrm{~d} \eta
$$

and hence can be treated with the same technique as in the proof of Theorem 1.10. But one prerequisite for discussing adjoints in the classical symbolic calculus (or in W. Hoh's symbolic calculus) is that first one needs to show that $p(x, D)$ is continuous from $\mathcal{S}\left(\mathbb{R}^{n}\right)$ to $\mathcal{S}\left(\mathbb{R}^{n}\right)$. This is not the case when working with non-smooth symbols. More precisely we find that

$$
|p(x, D) u|_{k, \mathcal{S}} \leq c_{k}|p|_{k}^{(m)}|u|_{m+2(n+1)+k, \mathcal{S}}
$$

holds for all $k \in \mathbb{N}$, where $|\cdot|_{k, \mathcal{S}}$ denotes the Schwartz space seminorm. But clearly, $|p|_{k}^{(m)}$ exists only for $k \leq \min \left\{M_{x}, M_{\xi}\right\}$. Using adjoints has the distinct advantage that we could show that tools like the (sharp) Gårding inequality or Friedrichs symmetrization are also available within our non-smooth symbolic calculus. Further investigations in this direction will be postponed to another paper. Instead, we present in the next section an easy way to identify compact pseudodifferential operators. This will allow us to use Fredholm theory to construct a parametrix.

\section{Parametrices and Feller semigroups}

We want to construct Feller semigroups, i.e., strongly continuous contraction semigroups on $C_{\infty}\left(\mathbb{R}^{n}\right)$ that are positivity preserving, by using the Hille-Yosida theorem and the symbolic calculus developed in section 1 . The novelty here is that we work with non-smooth symbols. Assume for the moment that $(A, D(A))$ extends 
to the generator of a Feller semigroup $\left(T_{t}\right)_{t \geq 0}$. A result from Ph. Courrège [3] states that if $C_{0}^{\infty}\left(\mathbb{R}^{n}\right) \subset D(A)$ then $A$ is on $C_{0}^{\infty}\left(\mathbb{R}^{n}\right)$ a pseudodifferential operator

$$
A u(x)=-q(x, D) u(x)=-(2 \pi)^{-\frac{n}{2}} \int_{\mathbb{R}^{n}} e^{i x \cdot \xi} q(x, \xi) \hat{u}(\xi) \mathrm{d} \xi,
$$

with symbol $q: \mathbb{R}^{n} \times \mathbb{R}^{n} \rightarrow \mathbb{C}$ such that $\xi \mapsto q(x, \xi)$ is continuous negative definite for all $x \in \mathbb{R}^{n}$. On the other hand, it was shown by N. Jacob [8], see also W. Hoh [6] in the context of a symbolic calculus, that starting with certain smooth negative definite symbols $q$, one can construct Feller semigroups by using the Hille-Yosida theorem, for details see also [11]. Since our symbolic calculus admits non-smooth symbols, we are going to assume from now on that we are given a symbol $p \in S_{\rho_{2}}^{2, \psi}\left(M_{x}, M_{\xi}\right)$ where $\psi$ is a continuous negative definite function of class $\Lambda^{\infty}, M_{x}, M_{\xi} \in \mathbb{N}_{0} \cup\{\infty\}$, and $\xi \mapsto p(x, \xi)$ is negative definite for all $x \in \mathbb{R}^{n}$. Note that every negative definite function is of order 2, compare Lemma 1.6. We want to show, under certain conditions on $M_{x}$ and $M_{\xi}$, that the corresponding pseudodifferential operator $-p(x, D)$ extends to the generator of a Feller semigroup. The Hille-Yosida theorem yields the desired result if the following conditions are satisfied:

$$
\begin{aligned}
& \text { - } D(-p(x, D)) \subset C_{\infty}\left(\mathbb{R}^{n}\right) \text { dense; } \\
& \text { - }-p(x, D) \text { is a dissipative operator; } \\
& \text { - } R(\lambda+p(x, D)) \text { is dense in } C_{\infty}\left(\mathbb{R}^{n}\right) \text { for some } \lambda>0 .
\end{aligned}
$$

The first two conditions are easily verified, it is the third condition that we have to work hardest for and for which we need the symbolic calculus developed in section 1 . The dissipativity of $-p(x, D)$ follows directly from the fact that it satisfies the positive maximum principle, see, e.g., S. Ethier and T. Kurtz [4]. Also, following the construction of Feller semigroups by W. Hoh [6] the anisotropic Sobolev spaces

$$
H^{s, \psi}\left(\mathbb{R}^{n}\right):=\left\{u \in \mathcal{S}^{\prime}\left(\mathbb{R}^{n}\right):\left\|(1+\psi(\cdot))^{\frac{s}{2}} \hat{u}\right\|_{L^{2}}<\infty\right\}
$$

are the right choice to handle the operator $-p(x, D)$. An embedding result, compare N. Jacob [11], yields that if $\psi(\xi) \geq c|\xi|^{r}$ for some $c>0, r>0$ and sufficiently large $|\xi|$, then for $s+2>\frac{n}{r}$ we have

$$
H^{s+2, \psi}\left(\mathbb{R}^{n}\right) \longleftrightarrow C_{\infty}\left(\mathbb{R}^{n}\right) .
$$

If we set $D(-p(x, D))=H^{s+2, \psi}\left(\mathbb{R}^{n}\right)$ then this gives (10).

As mentioned before, showing that (11) holds is non-trivial. It amounts to solving the equation

$$
(\lambda+p(x, D)) u=f
$$

where $f$ is an element of a dense subset of $C_{\infty}\left(\mathbb{R}^{n}\right)$ and $u \in D(-p(x, D))=$ $H^{s+2, \psi}\left(\mathbb{R}^{n}\right)$. Since $H^{s+2, \psi}\left(\mathbb{R}^{n}\right) \subset H^{s, \psi}\left(\mathbb{R}^{n}\right)$ and $-p(x, D)$ is an operator of order 2 
we suspect that we should choose $f \in H^{s, \psi}\left(\mathbb{R}^{n}\right)$. In order to justify this choice we need to prove that

$$
p(x, D): H^{s+2, \psi}\left(\mathbb{R}^{n}\right) \longrightarrow H^{s, \psi}\left(\mathbb{R}^{n}\right)
$$

is continuous. We need the Calderón-Vaillancourt theorem, see H. Kumano-go [13].

Theorem 2.1. Let $q: \mathbb{R}^{n} \times \mathbb{R}^{n} \rightarrow \mathbb{C}$ be a $C^{3,3}\left(\mathbb{R}^{2 n}\right)$-function such that

$$
\left|\partial_{\xi}^{\alpha} \partial_{x}^{\beta} q(x, \xi)\right| \leq c_{\alpha, \beta}
$$

Then

$$
\|q(x, D) u\|_{L^{2}} \leq c|q|_{3}^{(0)}\|u\|_{L^{2}}
$$

for some constant $c>0$.

Note that if we are given a symbol $q \in S_{\rho_{j}}^{0, \psi}\left(M_{x}, M_{\xi}\right)$ and $M_{x} \geq 3, M_{\xi} \geq 3$, then we may apply Theorem 2.1. Now we can prove the following result.

Theorem 2.2. Assume that $q \in S_{\rho_{j}}^{m, \psi}\left(M_{x}, M_{\xi}\right)$ where $m \in \mathbb{R}, M_{x}, M_{\xi} \in \mathbb{N}_{0} \cup\{\infty\}$, $j \in\{0,1,2\}$, and $\psi$ continuous negative definite symbol of class $\Lambda^{\infty}$. Then for $s \in \mathbb{R}$, if

$$
M_{x} \geq|s|+j+2 n+4 \quad \text { and } \quad M_{\xi} \geq 3
$$

we have that

$$
\|q(x, D) u\|_{H^{s, \psi}} \leq c\left|(1+\psi)^{\frac{s}{2}} \circ q \circ(1+\psi)^{\frac{-s-m}{2}}\right|_{3}^{(0)}\|u\|_{H^{s+m, \psi}},
$$

for all $u \in H^{s+m, \psi}\left(\mathbb{R}^{n}\right)$ and some $c>0$.

Proof. Since $\psi \in \Lambda^{\infty}$ we know that it is smooth, i.e.,

$$
(1+\psi(\cdot))^{\frac{s}{2}} \in S_{\rho_{j}}^{s, \psi}(\infty, \infty) .
$$

As $q \in S_{\rho_{j}}^{m, \psi}\left(M_{x}, M_{\xi}\right)$ we have by Theorem 1.10 (i) that, if $M_{x} \geq|s|+j+2 n+1$, then

$$
(1+\psi(\cdot))^{\frac{s}{2}} \circ q(\cdot, \cdot) \in S_{\rho_{j}}^{m+s, \psi}\left(M_{x}-|s|-j-2 n-1, M_{\xi}\right) .
$$

Also, since $(1+\psi(\cdot))^{\frac{-s-m}{2}}$ is a constant coefficient symbol, we find directly using Fourier transforms that

$$
(1+\psi(\cdot))^{\frac{s}{2}} \circ q(\cdot, \cdot) \circ(1+\psi(\cdot))^{\frac{-s-m}{2}} \in S_{\rho_{j}}^{0, \psi}\left(M_{x}-|s|-j-2 n-1, M_{\xi}\right) .
$$

By Theorem 2.1 we now find if

$$
M_{x}-|s|-j-2 n-1 \geq 3 \quad \text { and } \quad M_{\xi} \geq 3
$$

then

$$
\begin{aligned}
\|q(x, D) u\|_{H^{s, \psi}} & =\left\|(1+\psi(D))^{\frac{s}{2}} q(x, D) u\right\|_{L^{2}} \\
& =\left\|(1+\psi(D))^{\frac{s}{2}} q(x, D)(1+\psi(D))^{\frac{-s-m}{2}}\right\| L_{L^{2} \rightarrow L^{2}}\|u\|_{H^{s+m, \psi}} \\
& \leq\left|(1+\psi)^{\frac{s}{2}} \circ q \circ(1+\psi)^{\frac{-s-m}{2}}\right|_{3}^{(0)}\|u\|_{H^{s+m, \psi}} .
\end{aligned}
$$


It remains to solve equation (13), i.e., that for $f \in H^{s, \psi}\left(\mathbb{R}^{n}\right)$ there exists a solution $u \in H^{s+2, \psi}\left(\mathbb{R}^{n}\right)$ such that (10) holds. In order to achieve this we construct a parametrix using Fredholm theory. The starting point is the idea to use a special class of symbols that are asymptotically constant in the co-variable. We extend this idea that can be found, e.g., in V. V. Grushin [5] or H. Kumano-go and K. Taniguchi [14] to include our non-smooth negative definite symbols.

Definition 2.3. We denote by $S_{\infty, \rho_{j}}^{m, \psi}\left(M_{x}, M_{\xi}\right)$ the set of all symbols $q \in$ $S_{\rho_{j}}^{m, \psi}\left(M_{x}, M_{\xi}\right)$, such that for $|\alpha| \leq M_{\xi}$ and $|\beta| \leq M_{x}$,

$$
\left|\partial_{\xi}^{\alpha} \partial_{x}^{\beta} q(x, \xi)\right| \leq c_{\alpha, \beta}(x)(1+\psi(\xi))^{\frac{m-\rho_{j}(|\alpha|)}{2}}
$$

with $c_{\alpha, \beta} \in C_{\infty}\left(\mathbb{R}^{n}\right)$.

Cleary, we have that $S_{\infty, \rho_{j}}^{m, \psi}\left(M_{x}, M_{\xi}\right) \subset S_{\rho_{j}}^{m, \psi}\left(M_{x}, M_{\xi}\right)$, i.e. all results from previous sections remain valid. Note that such symbols are in the classical case sometimes called slowly varying symbols, and are denoted by $\dot{S}^{m}$ or $\widetilde{S}^{m}$ (compare, e.g., H. Kumano-go [13]). We choose here to follow V. V. Grushin's notation.

Lemma 2.4. Let $p \in S_{\rho_{j}}^{m, \psi}\left(M_{x}^{p}, M_{\xi}^{p}\right)$ and $q \in S_{\rho_{j}}^{l, \psi}\left(M_{x}^{q}, M_{\xi}^{q}\right)$ where $M_{\xi}^{p}$ and $M_{x}^{q}$ satisfy the conditions as given in Theorem 1.10 (ii) and $j \in\{1,2\}$. Furthermore let $\partial_{x}^{\beta} q \in S_{\infty, \rho_{j}}^{l, \psi}\left(M_{x}^{q}-1, M_{\xi}^{q}\right)$ for $|\beta|=1$. Then

$$
p \circ q(x, \xi)=p(x, \xi) q(x, \xi)+r(x, \xi)
$$

where $r \in S_{\infty, \rho_{j-1}}^{m+l-1, \psi}$.

Proof. The Lemma follows directly from Theorem 1.10 (ii) by looking at how the estimate of the remainder term is derived.

Lemma 2.5. Let $p: \mathbb{R}^{n} \times \mathbb{R}^{n} \rightarrow \mathbb{C}$ be a $C^{M_{x}, M_{\xi}}\left(\mathbb{R}^{2 n}\right)$-function, such that there exists a $r>0$ with $p(x, \xi)=0$ for all $(x, \xi) \in \mathbb{R}^{n} \times \mathbb{R}^{n}$ with $|x|>r$ or $|\xi|>r$. Then

$$
p(x, D): H^{s_{1}, \psi}\left(\mathbb{R}^{n}\right) \longrightarrow H^{s_{2}, \psi}\left(\mathbb{R}^{n}\right)
$$

is compact for all $s_{1}, s_{2} \in \mathbb{R}, s_{2} \leq M_{x}$, where $\psi$ is a continuous negative definite function of class $\Lambda^{\infty}$.

Proof. By Theorem 3.10.4 from N. Jacob [10] we know that

$$
H^{2 k}\left(\mathbb{R}^{n}\right) \hookrightarrow H^{2 k, \psi}\left(\mathbb{R}^{n}\right) \hookrightarrow H^{s_{2}, \psi}\left(\mathbb{R}^{n}\right)
$$

for $2 k \geq s_{2}$ since $\psi$ is a continuous negative definite function. Since the embedding is continuous it remains to show that $p(x, D): H^{s_{1}, \psi}\left(\mathbb{R}^{n}\right) \rightarrow H^{2 k}\left(\mathbb{R}^{n}\right)$ is compact, i.e., that the operator

$$
\left(1-\Delta_{x}\right)^{k} \circ p(x, D) \circ(1+\psi(D))^{-\frac{s_{1}}{2}}: L^{2}\left(\mathbb{R}^{n}\right) \longrightarrow L^{2}\left(\mathbb{R}^{n}\right)
$$


is compact. The symbol of $\Delta_{x}$ is $-|\xi|^{2}$ and as $\left(1+|\xi|^{2}\right)^{k}$ is a polynomial we know that $\left(1-\Delta_{x}\right)^{k}$ is a linear partial differential operator

$$
\left(1-\Delta_{x}\right)^{k}=\sum_{|\alpha| \leq 2 k} a_{\alpha} D_{x}^{\alpha}
$$

with constant coefficients. Then for $u \in \mathcal{S}\left(\mathbb{R}^{n}\right)$ and noting that $M_{x} \geq s_{2}$,

$$
\begin{aligned}
\left(1-\Delta_{x}\right)^{k} p(x, D)(1 & +\psi(D))^{-\frac{s_{1}}{2}} u(x) \\
& =(2 \pi)^{-\frac{n}{2}} \int_{\mathbb{R}^{n}} \sum_{|\alpha| \leq 2 k} a_{\alpha} D_{x}^{\alpha}\left(e^{i x \cdot \xi} p(x, \xi)\right)\left(1+|\xi|^{2}\right)^{-\frac{s_{1}}{2}} \hat{u}(\xi) \mathrm{d} \xi
\end{aligned}
$$

Using Leibniz' formula this gives us

$$
\left(1-\Delta_{x}\right)^{k} p(x, D)(1+\psi(D))^{-\frac{s_{1}}{2}} u(x)=\int_{\mathbb{R}^{n}} h(x, \xi) \hat{u}(\xi) \mathrm{d} \xi
$$

where $h$ is continuous and vanishes for $|x|>r$ or $|\xi|>r$. The Lemma now follows from the well-known fact that the integral operator

$$
L^{2}\left(B_{r}(0)\right) \ni v \longmapsto \int_{|y| \leq r} k(\cdot, y) v(y) \mathrm{d} y \in L^{2}\left(B_{r}(0)\right)
$$

is for a continuous kernel $k$ compact.

Note that Lemma 2.5 was proven without using Theorem 2.2. Now it is possible to show the following, compare V. V. Grushin [5] or H. Kumano-go [13, chapter 3, Theorem 5.14] for a similar technique.

Theorem 2.6. Let $q \in S_{\infty, \rho_{j}}^{m, \psi_{j}}\left(M_{x}, M_{\xi}\right), s \in \mathbb{R}$, and

$$
\begin{aligned}
& M_{x} \geq|s|+j+2 n+4 \\
& M_{\xi} \geq 3 .
\end{aligned}
$$

Then for every $\varepsilon>0$

$$
q(x, D): H^{s+m, \psi}\left(\mathbb{R}^{n}\right) \longrightarrow H^{s-\varepsilon, \psi}\left(\mathbb{R}^{n}\right)
$$

is compact.

Proof. Let $\varphi \in C_{0}^{\infty}\left(\mathbb{R}^{n}\right)$ such that $\varphi(x)=0$ for $|x|>1$ and $\varphi(x)=1$ for $|x| \leq \frac{1}{2}$. We set

$$
q_{s}(x, D):=(1+\psi(D))^{\frac{s-\varepsilon}{2}} \circ q(x, D) \circ(1+\psi(D))^{\frac{-s-m}{2}} .
$$

The operator

$$
\varphi\left(\frac{x}{l}\right) \circ q_{s}(x, D) \circ \varphi\left(\frac{D}{l}\right): L^{2}\left(\mathbb{R}^{n}\right) \longrightarrow L^{2}\left(\mathbb{R}^{n}\right)
$$


has symbol

$$
\varphi\left(\frac{x}{l}\right) q_{s}(x, \xi) \varphi\left(\frac{\xi}{l}\right)
$$

and hence is by Lemma 2.5 compact. It follows that

$$
\left\|q_{s}(x, D)-\varphi\left(\frac{x}{l}\right) \circ q_{s}(x, D) \circ \varphi\left(\frac{D}{l}\right)\right\|_{L^{2} \rightarrow L^{2}} \longrightarrow 0
$$

since $\left(1-\varphi\left(\frac{x}{l}\right) \varphi\left(\frac{\xi}{l}\right)\right) q_{s}(x, \xi) \rightarrow 0$ in $S_{\rho_{j}}^{0, \psi}$ for $l \rightarrow \infty$. As the limit of a (with respect to the operator norm) convergent sequence of compact operators is again compact, we conclude that $q_{s}(x, D): L^{2}\left(\mathbb{R}^{n}\right) \rightarrow L^{2}\left(\mathbb{R}^{n}\right)$ is compact. Now we write $q(x, D)$ as

$$
q(x, D)=(1+\psi(D))^{\frac{-s+\varepsilon}{2}} \circ q_{s}(x, D) \circ(1+\psi(D))^{\frac{s+m}{2}}
$$

and find that $q(x, D): H^{s+m, \psi}\left(\mathbb{R}^{n}\right) \rightarrow H^{s-\varepsilon, \psi}\left(\mathbb{R}^{n}\right)$ is compact.

We need the following result from functional analysis (see, e.g., K. Yosida [18]).

Theorem 2.7. Let $T \in \mathcal{L}(E, F)$, where $E, F$ are normed spaces and $\mathcal{L}(E, F)$ denotes the set of all continuous linear operators from $E$ into $F$. Then $T$ is a Fredholm operator if and only if there exist $S_{1}, S_{2} \in \mathcal{L}(F, E)$ with

$$
T S_{1}=\operatorname{Id}_{F}+K_{1} \quad \text { and } \quad S_{2} T=\operatorname{Id}_{E}+K_{2},
$$

where $K_{1}$ and $K_{2}$ are compact.

Now we are in a position to prove the main theorem, for classical parametrix constructions (see, e.g., K. Yosida [18]). We point out that we restrict ourselves to the case where the order of the symbol is 2 since this is the case of interest to us, compare Theorem 2.9. It is nevertheless possible to construct a parametrix also in the general case.

Theorem 2.8. Let $p \in S_{\rho_{2}}^{2, \psi}\left(M_{x}, M_{\xi}\right)$, such that $\partial_{x}^{\beta} p \in S_{\infty, \rho_{2}}^{2, \psi}\left(M_{x}-1, M_{\xi}\right)$ for all $|\beta|=1$. Also assume that there exists $r>0$ and $\delta>0$ such that

$$
|p(x, \xi)| \geq \delta(1+\psi(\xi))
$$

for all $|x|^{2}+|\xi|^{2} \geq r^{2}$. Then for $s \in \mathbb{R}$, if

$$
M_{x} \geq|s|+4 n+12
$$

and

$$
M_{\xi} \geq 2 n+9
$$

we find that

$$
p(x, D): H^{s+2, \psi}\left(\mathbb{R}^{n}\right) \longrightarrow H^{s, \psi}\left(\mathbb{R}^{n}\right)
$$

is a Fredholm operator. 
Proof. If $M_{x} \geq|s|+2 n+4$ and $M_{\xi} \geq 3$ we have by Theorem 2.2 that $p(x, D): H^{s+2, \psi}\left(\mathbb{R}^{n}\right) \rightarrow H^{s, \psi}\left(\mathbb{R}^{n}\right)$. It remains to show that $p(x, D)$ is a Fredholm operator.

Let $\varphi \in C^{\infty}\left(\mathbb{R}^{n} \times \mathbb{R}^{n}\right)$ with $\varphi(x, \xi)=0$ for $|x|^{2}+|\xi|^{2}<r^{2}$ and $\varphi(x, \xi)=1$ for $|x|^{2}+|\xi|^{2} \geq 2 r^{2}$. If we set

$$
q(x, \xi):= \begin{cases}\varphi(x, \xi) p(x, \xi)^{-1}, & \text { if }|x|^{2}+|\xi|^{2} \geq r^{2} \\ 0, & \text { if }|x|^{2}+|\xi|^{2}<r^{2}\end{cases}
$$

then $q \in S_{\rho_{2}}^{-2, \psi}\left(M_{x}, M_{\xi}\right)$ and $\partial_{x}^{\beta} q \in S_{\infty, \rho_{2}}^{-2, \psi}\left(M_{x}-1, M_{\xi}\right)$ for $|\beta|=1$ as is easily seen using Leibniz' formula and the chain rule (note that for a differentiable and pointwise invertible matrix-valued function $a$ we have that $\left.\left(\partial_{x_{j}} a^{-1}\right)=-a^{-1}\left(\partial_{x_{j}} a\right) a^{-1}\right)$. It holds that

$$
p(x, \xi) q(x, \xi)=q(x, \xi) p(x, \xi)= \begin{cases}\varphi(x, \xi), & \text { if }|x|^{2}+|\xi|^{2} \geq r^{2} \\ 0, & \text { if }|x|^{2}+|\xi|^{2}<r^{2}\end{cases}
$$

We now assume that $|x|^{2}+|\xi|^{2} \geq r^{2}$. We want to use Theorem 1.10 (ii) and hence need to check that the differentiability conditions are satisfied. We find that

$$
(q \circ p)(x, \xi)=\varphi(x, \xi)+r_{1}(x, \xi) \quad \text { and } \quad(p \circ q)(x, \xi)=\varphi(x, \xi)+r_{2}(x, \xi)
$$

if

$$
\begin{aligned}
& M_{x} \geq|-2-1|+2+2 n+1=6+2 n, \\
& M_{\xi} \geq 6+2 n .
\end{aligned}
$$

Here, $r_{1}, r_{2} \in S_{\infty, \rho_{1}}^{-1, \psi}$, see Lemma 2.4 , and

$$
\begin{aligned}
& M_{x}^{r_{1}}=M_{x}^{r_{2}}=M_{x}-6-2 n, \\
& M_{\xi}^{r_{1}}=M_{\xi}^{r_{2}}=M_{\xi}-6-2 n .
\end{aligned}
$$

Since $1-\varphi(x, \xi) \in C_{0}^{\infty}\left(\mathbb{R}^{n} \times \mathbb{R}^{n}\right)$ we have that $1-\varphi \in S_{\infty, \rho_{1}}^{-1, \psi}(\infty, \infty)$ and thus we may write (15) as

$$
\begin{aligned}
& (q \circ p)(x, \xi)=-1+(1-\varphi(x, \xi))+r_{1}(x, \xi), \\
& (p \circ q)(x, \xi)=-1+(1-\varphi(x, \xi))+r_{2}(x, \xi) .
\end{aligned}
$$

Hence we find for the corresponding pseudodifferential operators that

$$
q(x, D) \circ p(x, D)=\operatorname{Id}+R_{1}^{\prime}(x, D) \text { and } p(x, D) \circ q(x, D)=\operatorname{Id}+R_{2}^{\prime}(x, D),
$$

where $r_{1}^{\prime}, r_{2}^{\prime} \in S_{\infty, \rho_{1}}^{-1, \psi}$ and $M_{x}^{r_{1}^{\prime}}=M_{x}^{r_{1}}, M_{\xi}^{r_{1}^{\prime}}=M_{\xi}^{r_{1}}, M_{x}^{r_{2}^{\prime}}=M_{x}^{r_{2}}$, and $M_{\xi}^{r_{2}^{\prime}}=M_{\xi}^{r_{2}}$. We also have by Theorem 2.2 that if

$$
M_{x} \geq|s|+2 n+6 \text { and } M_{\xi} \geq 3
$$


then $q(x, D): H^{s-2, \psi}\left(\mathbb{R}^{n}\right) \rightarrow H^{s, \psi}\left(\mathbb{R}^{n}\right)$. Hence

$$
\begin{aligned}
& p(x, D) q(x, D): H^{s-2, \psi}\left(\mathbb{R}^{n}\right) \longrightarrow H^{s-2, \psi}\left(\mathbb{R}^{n}\right), \\
& q(x, D) p(x, D): \quad H^{s, \psi}\left(\mathbb{R}^{n}\right) \longrightarrow H^{s, \psi}\left(\mathbb{R}^{n}\right) .
\end{aligned}
$$

In order to apply Theorem 2.7 it remains to show that

$$
R_{1}^{\prime}(x, D): H^{s-2, \psi}\left(\mathbb{R}^{n}\right) \longrightarrow H^{s-2, \psi}\left(\mathbb{R}^{n}\right)
$$

and

$$
R_{2}^{\prime}(x, D): \quad H^{s, \psi}\left(\mathbb{R}^{n}\right) \longrightarrow H^{s, \psi}\left(\mathbb{R}^{n}\right)
$$

are compact. The compactness and the mapping properties of $R_{1}^{\prime}(x, D)$ and $R_{2}^{\prime}(x, D)$ follow from Theorem 2.6 if

$$
M_{x}^{r_{1}^{\prime}}=M_{x}^{r_{2}^{\prime}} \geq|s|+2 n+6 \quad \text { and } \quad M_{\xi}^{r_{1}^{\prime}}=M_{\xi}^{r_{2}^{\prime}} \geq 3 .
$$

But this means that we need

$$
M_{x} \geq|s|+4 n+12 \text { and } M_{\xi} \geq 2 n+9 .
$$

Using Theorem 2.7 we can now solve equation (12) and arrive at the following theorem.

Theorem 2.9. Let $p \in S_{\rho_{2}}^{2, \psi}\left(M_{x}, M_{\xi}\right), \psi \in \Lambda^{\infty}$, be given such that $\xi \mapsto p(x, \xi)$ is continuous negative definite for all $x \in \mathbb{R}^{n}$ and $\partial_{x}^{\beta} p \in S_{\infty, \rho_{2}}^{2, \psi}\left(M_{x}-1, M_{\xi}\right)$ for $|\beta|=1$. Assume further that $\psi(\xi) \geq c|\xi|^{r}$ for some $c>0, r>0$,

$$
M_{x} \geq\left|\frac{n}{r}-1\right|+4 n+12, \quad M_{\xi} \geq 2 n+9
$$

and that there exists a $\delta>0$ such that

$$
|p(x, \xi)| \geq \delta(1+\psi(\xi))
$$

for large $\xi$ and $x \in \mathbb{R}^{n}$. Then the corresponding pseudodifferential operator $-p(x, D)$ extends to the generator of a Feller semigroup $\left(T_{t}\right)_{t \geq 0}$.

Proof. Following the discussion at the beginning of this section, it remains to solve (13) We already know that $H^{s+2, \psi}\left(\mathbb{R}^{n}\right) \hookrightarrow C_{\infty}\left(\mathbb{R}^{n}\right)$ for $s>\frac{n}{r}-2$ where $\psi(\xi) \geq c|\xi|^{r}$. Since $r>0$ is small we can assume without loss of generality that $s>0$ is large. Furthermore, as $\xi \mapsto p(x, \xi)$ is negative definite we have that $p(x, 0)=0$ for all $x \in \mathbb{R}^{n}$. On the other hand, Theorem 2.8 requires the symbol to satisfy (14). Hence we consider the operator $p(x, D)+\delta$ with symbol $p(x, \xi)+\delta$ and find that this symbol satisfies (14). By setting $s:=\frac{n}{r}-1$ and using Theorem 2.8 we now get that 
$p(x, D)+\delta$ is a Fredholm operator. Hence we can use Theorem 2.7 and reduce solving the equation $\lambda u+p(x, D) u=f$ to solving the equation $\frac{1}{\lambda} u+K u=S f$ where $K$ is compact. Using the Fredholm alternative we either find that this equation has a unique solution for all $f$, and in this case we are done - or, that this equation does not have a solution for $\lambda \in M$, where $M$ is some countable subset of the real numbers. In this case we simply choose $\lambda>0$ in such a way that it does not belong to $M$ since it suffices to solve the equation $\lambda u+p(x, D) u=f$ only for one arbitrary $\lambda>0$. An application of the Hille-Yosida theorem now yields the result.

The condition on $M_{x}$ in the above Theorem can in practice be read as $M_{x}=\infty$ since $r>0$ small. Compared to W. Hoh's construction of Feller semigroups that are pregenerated by pseudodifferential operators with negative definite symbols, compare [6], let us make some remarks: Our approach admits non-smooth symbols whereas other constructions require smooth symbols. In particular, if we set in Theorem 2.9, $M_{x}=\infty$ and $M_{\xi}=\infty$, and if we take away the additional restriction that the symbol has to be asymptotically constant, our result in Theorem 2.9 coincides with the result of W. Hoh. Note that our result simplifies the construction since tools such as the Gårding inequality, Friedrichs mollifier and variational solutions, are not needed.

\section{Final remarks}

The differentiability conditions found in Theorem 2.9 can in principle be tracked back to the fact that we use the oscillatory integral representation of the symbol of the composition of two pseudodifferential operators. It is a subject of further study whether it is possible to use the singular integral representation and direct estimates on the kernel to weaken these conditions. As mentioned before, it might also be interesting to consider the adjoint representation of a pseudodifferential operator with non-smooth symbol to develop further tools such as the Gårding inequality. This would enable to us to extend the final result in such a way that we could also include symbols that are not asymptotically constant in the co-variable.

Acknowledgement. The author would like to thank N. Jacob for many helpful discussions.

\section{References}

[1] S. Alinhac and P. Gérard, Opérateurs pseudo-différentiels et théorème de Nash-Moser, Savoirs Actuels, InterEditions, Éditions du Centre National de la Recherche Scientifique (CNRS), Meudon, Paris, 1991.

[2] A.-P. Calderón and R. Vaillancourt, On the boundedness of pseudo-differential operators, J. Math. Soc. Japan 23 (1971), 374-378. 
[3] Ph. Courrège, Sur la forme intégro-différentielle des opérateurs de $C_{K}^{\infty}$ dans $C$ satisfaisant au principe du maximum, Sém. Théorié du Potentiel (1965/66). Exposé 2, 38 pp.

[4] S. N. Ethier and T. G. Kurtz, Markov processes: Characterization and convergence, Wiley Series in Probability and Mathematical Statistics, John Wiley \& Sons Inc., New York, 1986.

[5] V. V. Grushin, Pseudodifferential operators on $\mathbb{R}^{n}$ with bounded symbols, Funkt. Anal. Appl. 4 (1970), 202-212.

[6] W. Hoh, A symbolic calculus for pseudo-differential operators generating Feller semigroups, Osaka J. Math. 35 (1998), no. 4, 789-820.

[7] L. Hörmander, The analysis of linear partial differential operators, IV, Grundlehren der Mathematischen Wissenschaften, vol. 275, Springer-Verlag, Berlin, 1985. Fourier integral operators.

[8] N. Jacob, Feller semigroups, Dirichlet forms, and pseudodifferential operators, Forum Math. 4 (1992), no. 5, 433-446.

[9] Characteristic functions and symbols in the theory of Feller processes, Potential Anal. 8 (1998), no. 1, 61-68.

[10] _ Pseudo differential operators and Markov processes, I: Fourier analysis and semigroups, Imperial College Press, London, 2001. Reprinted in 2006.

[11] __ Pseudo differential operators and Markov processes, II: Generators and their potential theory, Imperial College Press, London, 2002.

[12] _ Pseudo differential operators and Markov processes, III: Markov processes and applications, Imperial College Press, London, 2005.

[13] H. Kumano-go, Pseudodifferential operators, MIT Press, Cambridge, Mass., 1981.

[14] H. Kumano-go and K. Taniguchi, Fourier integral operators of multiphase and the fundamental solution for a hyperbolic system, Funkcial. Ekvac. 22 (1979), no. 2, 161-196.

[15] R. L. Schilling, Conservativeness of semigroups generated by pseudo-differential operators, Potential Anal. 9 (1998), no. 1, 91-104.

[16] E. M. Stein, Harmonic analysis: Real-variable methods, orthogonality, and oscillatory integrals, Princeton Mathematical Series, vol. 43, Princeton University Press, Princeton, NJ, 1993.

[17] M. E. Taylor, Tools for PDE: Pseudodifferential operators, paradifferential operators and layer potentials, Mathematical Surveys and Monographs, vol. 81, American Mathematical Society, Providence, RI, 2000.

[18] K. Yosida, Functional analysis, 2nd ed., Grundlehren der Mathematischen Wissenschaften, vol. 123, Springer-Verlag, Berlin, 1971 